\title{
Application of bench studies at the bedside to improve outcomes in the management of severe diabetic ketoacidosis in children - a narrative review
}

\author{
Namita Ravikumar, Arun Bansal^ \\ Division of Pediatric Critical Care, Department of Paediatrics, Advanced Paediatrics Centre, Postgraduate Institute of Medical Education and \\ Research (PGIMER), Chandigarh, India \\ Contributions: (I) Conception and design: Both authors; (II) Administrative support: A Bansal; (III) Provision of study materials or patients: N \\ Ravikumar; (IV) Collection and assembly of data: N Ravikumar; (V) Data analysis and interpretation: Both authors; (VI) Manuscript writing: Both \\ authors; (VII) Final approval of manuscript: Both authors. \\ Correspondence to: Dr. Arun Bansal. Professor, Department of Pediatrics, Advanced Pediatrics Centre, Postgraduate Institute of Medical Education \\ and Research (PGIMER), Chandigarh 160012, India. Email: drarunbansal@gmail.com.
}

\begin{abstract}
Objective: In this review, we address a few key issues and the challenges faced in the management of severe diabetic ketoacidosis (DKA) in children, highlighting the existing standard of care, supported by evidence and bench studies.

Background: The classic triad of DKA namely hyperglycemia, metabolic acidosis and ketonemia warrants immediate attention with fluids and insulin. Correction of dehydration in DKA is of utmost priority and the calculation of fluid volume and choice of fluid have remained a matter of debate. Insulin therapy, to halt the ketone production, in DKA has undergone wide variations in dose and preparation since its discovery. Although the mortality due to severe DKA has remarkably decreased, complications like cerebral edema and acute kidney injury (AKI) continue to haunt the intensivists and endocrinologists on a few occasions.

Methods: We have selected a few important questions in the management of severe DKA in children, addressing the challenges, reviewing the studies, guidelines and bedside practices with evidence in this narrative review.

Conclusions: The focus of management should be to understand and normalise the deranged physiology rather than trying to get normal laboratory reports. This needs careful understanding of the pathogenesis and deriving conclusion from bench studies. With newer studies and evidence, guidelines are revised every few years. There is a trend towards more conservative therapy, with continuous and advanced monitoring. Switching to subcutaneous insulin and oral hydration is done as early as possible with clinical monitoring and resolution of DKA. Management of severe DKA in children can vary from simple fluid titration and insulin infusion in mild cases to a scenario with multiorgan dysfunction requiring intensive monitoring and advanced organ support. Individualisation of therapy to suit the needs with the available evidence and expertise is extremely essential.
\end{abstract}

Keywords: Diabetic ketoacidosis (DKA); insulin; hyperglycemia; cerebral edema

Submitted Jan 06, 2021. Accepted for publication May 14, 2021.

doi: $10.21037 /$ tp-21-5

View this article at: http://dx.doi.org/10.21037/tp-21-5

^ ORCID: 0000-0001-6212-6889. 


\section{Introduction}

Diabetic ketoacidosis (DKA) is a life threatening emergency manifesting with hyperglycemia (RBS $>200 \mathrm{mg} / \mathrm{dL}$ ), high anion gap metabolic acidosis $\left(\mathrm{pH}<7.3\right.$ and/or $\mathrm{HCO}_{3}$ $<15 \mathrm{mmol} / \mathrm{L}$ ) and ketonemia (Beta-hydroxybutyrate $>3$ ) (1). Management principles include replacement of fluid deficit, supplementation of insulin and prevention of complications like cerebral edema, electrolyte disturbance and acute kidney injury (AKI) (2). The greatest breakthrough in the management of DKA came with discovery of Insulin therapy, which has resulted in decreased mortality. The dosage range has undergone modifications through the years based on new evidence. From 1923 to 1940, high dose insulin treatment was the norm with minimal focus on fluid therapy (3). Likewise, a lot of debate regarding choice and volume of fluid has led to change of management protocols with slight variations among the various guidelines published by different societies $(1,4,5)$. Low dose insulin infusion with aggressive fluid resuscitation began in 1972 and the trend nowadays is towards a more conservative fluid therapy (3). Delay in identification, inadequate pre-referral management and development of complications like cerebral edema and AKI still remain the leading causes of mortality in children with newly diagnosed type 1 Diabetes mellitus, especially in developing countries. Frequent monitoring of blood gas, blood glucose and electrolytes for adequate titration of fluid, insulin and glucose content of the fluid forms the core of DKA management (6). We present the following article in accordance with the Narrative Review reporting checklist (available at http://dx.doi.org/10.21037/tp-21-5).

\section{Objectives}

We have selected a few important questions in the management of severe DKA in children addressing the challenges faced at bedside.

\section{Methods}

We have reviewed the studies, guidelines and bedside practices over the past 20 years with evidence in this narrative review addressing a few challenges faced at bedside.

\section{Discussion (Table 1)}

\section{Fluid volume-is there a way to estimate the ideal amount?}

\section{Challenges}

Clinical estimation of the degree of dehydration in DKA is unreliable due to the preservation of intravascular volume till the end stages, secondary to hyperosmolarity. Metabolic acidosis leading to drying of the mucosa further hampers the assessment. The need for fluid bolus in the first hour in all children with DKA has undergone revision in the recent recommendations.

\section{Studies}

Many studies have evaluated ways to estimate the actual degree of dehydration; as both under- and overestimation are detrimental. Degree of clinical dehydration, severity of DKA, serial weight assessment and blood urea values have been used to calculate the deficit fluid replacement. Koves et al. found no agreement between the clinical assessment of dehydration by two independent observers and the dehydration measured by weight as the dehydration was overestimated in $24 \%$ and underestimated in $46 \%$ of cases in clinical assessment group $(\kappa=0.05)(7)$.

Table 1 Recommendations on key issues in the management of severe DKA in children

\begin{tabular}{ll}
\hline Key management issues & Recommendations based on evidence \\
\hline Fluid volume estimation & $6-7 \%$ dehydration correction \\
Fluid choice & $0.9 \%$ normal saline. Consider balanced crystalloids, if risk of acute kidney injury \\
Insulin dose & $0.05-0.1 \mathrm{U} / \mathrm{kg} / \mathrm{h}$, as per clinical assessment \\
Cerebral edema etiology & Related to severity at onset than therapy related \\
Management of cerebral edema & Hyperosmolar therapy, delayed intubation, controlled hyperventilation \\
DKA resolution detection & Anion gap closure, normalization of blood ketones by POC test \\
\hline
\end{tabular}

DKA, diabetic ketoacidosis; POC, point of care. 


\section{Bedside}

Fluid bolus of isotonic saline $10-20 \mathrm{~mL} / \mathrm{kg}$ over 30 60 minutes is recommended in the presence of poor perfusion and bolus volume exceeding $20 \mathrm{~mL} / \mathrm{kg}$ has to be subtracted from the calculated dehydration correction volume (8). Most studies have concluded that a fluid replacement of $6-7 \%$ reliably corrects dehydration regardless of the severity of DKA $(7,9)$. In order to titrate the fluid, sodium and glucose requirement and avoid rapid change of shifts in osmolarity, a two-bag system of fluid infusion has been proposed. Though time to resolution and blood glucose values were comparable, this system facilitates faster action to changing glucose concentrations in an emergency $(10,11)$. The Pediatric Emergency Care Applied Research Network (PECARN) DKA fluid group compared fast ( $10 \%$ of body weight) versus slow ( $5 \%$ body weight) administration of $0.9 \%$ and $0.45 \%$ saline and found no significant difference in the neurological outcomes in children with DKA, although the cohort predominantly constituted mild cases (12).

\section{Fluid choice-can we prevent AKI?}

\section{Challenges}

Crystalloids, mainly normal saline (NS) and Ringer's Lactate, remain the first choice to combat dehydration in DKA (8). Hyperchloremic normal anion gap metabolic acidosis often appears during resolution. High chloride content in NS, AKI and preferential ketone excretion are the likely explanations. The cause effect relationship of hyperchloremia and AKI has also been postulated. The question of whether balanced crystalloids are preferred over unbalanced fluids remains elusive.

\section{Studies}

Animal studies have shown that perfusions with high chloride concentrations enhanced the pressor effects of vasoconstrictor agents (13). The use of balanced electrolyte solution was associated with lower serum chloride levels and higher bicarbonate levels in the post-resuscitation phase in adults with DKA (14). The presence of hyperchloremia at 24 hours of admission was an independent predictor for AKI, however, further studies are needed to establish causeeffect relationship (15).

\section{Bedside}

SPinK trial by Williams et al. comparing $0.9 \%$ saline with plasmalyte in children with DKA did not show a significant difference in AKI or DKA resolution (16). Yung and colleagues noted that Hartmann Solution may be used in children as an initial choice of fluid in children with DKA with some added benefit in those with severe DKA in terms of time to resolution (17). Till further studies, it is preferred to use isotonic crystalloids routinely and need for balanced crystalloids on a case to case basis may be assessed in children with high risk for AKI.

\section{Insulin dose- is hyperglycemia or bypoglycemia more dangerous?}

\section{Challenges}

DKA is a state of insulin deficiency with an excess of counter-regulatory hormones. Insulin therapy for DKA is usually initiated after an hour of fluid resuscitation; as the correction of dehydration also leads to fall in blood glucose levels (1). Standard dosage regime is the use of rapid acting insulin at an infusion of $0.1 \mathrm{U} / \mathrm{kg} / \mathrm{hour}$. With rehydration and insulin therapy, it is expected that the blood glucose will fall smoothly along with slow reduction of serum osmolarity. However, it is often seen that with therapy there is a precipitous fall of blood glucose requiring initiation of high glucose containing fluids to balance the hypoglycemia and ketosis (18).

\section{Studies}

Bradley et al. found more proportion of hours (24\%) with a fall in serum glucose greater than $100 \mathrm{mg} / \mathrm{dL}$ per hour during infusion rates of insulin at $0.1 \mathrm{U} / \mathrm{kg} / \mathrm{h}$ as compared to the insulin at $0.05 \mathrm{U} / \mathrm{kg} / \mathrm{h}(4.8 \%)$ (19). Likewise the use of bolus dose insulin in the older regimes also had more complications with no added benefit. The trajectories of glucose-corrected sodium and effective osmolarity are helpful in predicting the onset of cerebral edema (20). However, in this retrospective study by Durward et al. there were few confounders like the use of hyperosmolar therapy and the effect of treatment received prior to referral (20). Also, they had higher proportion of severe DKA, among children with cerebral edema, who received more fluids in the first 4 hours.

\section{Bedside}

Gradual reduction of glucose along with effective osmolarity and rise of sodium was noted in the children receiving $0.05 \mathrm{U} / \mathrm{kg} / \mathrm{h}$ with similar time to DKA resolution in a study by Al Hanshi et al. (21). In the study by Nallasamy et al., low dose insulin regime was found to be 
non-inferior to standard dose in terms of blood glucose reduction and resolution of acidosis (22). Hypoglycemia and hypokalemia episodes were noted more in the standard group of insulin at $0.1 \mathrm{U} / \mathrm{kg} / \mathrm{h}$. These were more prominent in the malnourished cohort. The International Society for Pediatric and Adolescent Diabetes (ISPAD) guidelines have incorporated these landmark studies suggesting a dosage range of $0.05-0.1$ units $/ \mathrm{kg} /$ hour (1).

\section{Cerebral edema-is the damage already done or are we contributing to it?}

\section{Challenges}

Cerebral edema is seen in about $0.5-1 \%$ of patients and contributes to a significant neurological morbidity. Overt raised intracranial pressure (ICP) is rare. However, neuroimaging and optic nerve sheath diameter changes in children with severe DKA has shown that subtle cerebral edema can be missed clinically and can lead to neurological deficits $(23,24)$.

\section{Studies}

The pathogenesis of cerebral edema is still evolving and the incidence of pre-treatment cerebral edema suggests that the contribution from fluid management is lesser than thought previously (25). Severe DKA with untreated hypoperfusion, degree of acidosis, hypocapnia compensatory to severe metabolic acidosis and raised blood urea cause vasoconstriction and lead to reduced cerebral perfusion. An increase in blood brain barrier (BBB) permeability precipitates vasogenic cerebral edema with secondary worsening after start of fluid therapy due to reperfusion injury mediated cytotoxic edema (8). Hypothesis regarding the role of Interleukins 1 and 6, released by inflammasomes in the pathogenesis of both vasogenic and cytotoxic cerebral edema has been generated $(26,27)$. Alterations in matrix metalloprotein (MMP) expression are found to mediate BBB dysfunction in inflammatory states and lower circulating MMP-2 levels with high MMP-9 levels are seen in children during DKA (28).

\section{Bedside}

The PECARN DKA fluid group studied the effect of fluid infusion rates and content of sodium chloride and concluded that neither influenced the neurological outcome in children (12). Studies with correlation between Brain Magnetic resonance imaging (MRI) findings, markers of brain injury and interleukin levels may pave the way for better understanding of the pathophysiology and therapeutic options for cerebral edema in DKA.

\section{Hyperventilating and comatose-when to intubate?}

\section{Challenges}

The validity of Glasgow coma scale (GCS) for assessment and monitoring for cerebral edema in DKA is questionable. Considering various pathogenic mechanisms for cerebral edema, the role of hyperosmolar therapy in its management and the choice between mannitol and hypertonic saline are extrapolated from other etiologies of raised ICP like traumatic brain injury (29). The decision and timing of intubation and controlled ventilation in severe DKA is challenging as it takes away the body's compensatory responses and has the potential to lead to fluctuations in partial pressures of carbon dioxide $\left(\mathrm{paCO}_{2}\right)$ in blood.

\section{Studies}

Computerised Tomography (CT) of the brain, magnetic resonance sequences like diffusion weighted MRI, diffusion tensor imaging (DTI) parameters, apparent diffusion coefficient (ADC), fractional anisotropy (FA), and radial and axial diffusivity to study changes in white matter microstructure have been used to detect subclinical cerebral abnormalities in children with DKA (30). Increasing trend towards the use of hypertonic saline compared to mannitol in DKA associated cerebral edema has been seen mirroring the change in other etiologies of raised ICP. A retrospective analysis found the use of hypertonic saline to be associated with higher mortality in children with symptomatic cerebral edema in DKA (31). In children with severe metabolic acidosis with hypocapnia, intubation and mechanical ventilation targeting a "normal' $\mathrm{paCO}_{2}$ may be detrimental as the $\mathrm{pH}$ of $\mathrm{CSF}$ has a rapid response to changing $\mathrm{CO}_{2}$ and may worsen CSF $\mathrm{pH}$. This phenomenon has been well described with the use of sodium bicarbonate in DKA leading to paradoxical CNS acidosis. It is best to target a slightly lower $\mathrm{paCO}_{2}$ in children who developed low serum $\mathrm{HCO}_{3}(32)$.

\section{Bedside}

In children with raised ICP secondary to acute CNS infection, $3 \%$ hypertonic saline was found to be better than $20 \%$ mannitol in control of invasively monitored ICP (33). In these children, the major contributory factors for diffuse cerebral edema included inflammatory, vasogenic, or cytotoxic edema due to various stages of 
illness based on bacterial or viral etiology. In addition, this study by Rameshkumar et al. found more chances of rebound increase in ICP, therapy related hypotension and higher need for hyperventilation to manage ICP spikes in the mannitol group. Although no clear guidelines or studies are available comparing mannitol versus hypertonic saline in DKA associated cerebral edema, serial monitoring for changes in sodium and effective osmolarity is essential regardless of the osmotherapy used. Intubation and mechanical ventilation are best avoided until a point of exhaustion to avoid distortion of the adaptive physiology. And when controlled ventilation has been initiated, target $\mathrm{PaCO}_{2}$ for the level of $\mathrm{HCO}_{3}$ prior to intubation may be calculated and strictly monitored to avoid hyperemia related to rise in $\mathrm{CO}_{2}$ secondary to sedation or depressed sensorium. Care should also be taken to avoid aggressive hyperventilation.

\section{Measurement of blood ketones—closing the gap}

\section{Challenges}

Urine ketones may herald the development of DKA, however, with ongoing treatment, ketones tend to be excreted in urine beyond recovery. Also, acetoacetate is the ketone detected on urine dipstick whereas beta-hydroxy butyrate (BOHB) remains the predominant blood ketone in DKA. Resolution of DKA is determined by the normalization of $\mathrm{pH} \geq 7.3$ and serum bicarbonate $\left(\mathrm{HCO}_{3} \geq 15\right)$. However, the appearance of hyperchloremia during DKA resolution and accompanying AKI can mask the resolution due to the co-existence of normal anion gap metabolic acidosis. The importance of differentiating this from non-resolution can have consequences in the further management.

\section{Studies}

The closure of anion gap can suggest resolution of DKA in the situation of persistent metabolic acidosis. Measurement of blood ketone is for the confirmation of resolution of DKA and several point of care (POC) tests to determine BOHB have been developed.

\section{Bedside}

With advances in technology, clinical decisions can be based on hand held, POC tests to measure blood ketone levels (34). They aid in diagnosis, determination of severity and confirm resolution especially in the presence of hyperchloremic normal anion gap metabolic acidosis. A study by Kurup et al. in children with DKA found excellent correlation between POC and serum BOHB measurements (35).

\section{Conclusions}

To summarise, the initial fluid volume for rehydration is $6-7 \%$ with maintenance fluid along with further monitoring and titration. The choice of fluid remains $0.9 \%$ normal saline with a switch to balanced salt solutions in children with severe DKA and high risk of AKI. Insulin infusion is initiated after one hour of fluids at $0.05-$ 0.1 units $/ \mathrm{kg} /$ hour as per clinical assessment and titration done based on response. Early and appropriate therapy minimizes the complications like cerebral edema, AKI and electrolyte abnormalities. Although the incidence of cerebral edema has decreased in the recent times, the onset seems to be prior to start of therapy and related to severity of DKA than therapy related cerebral edema. A small subset of children may require assisted ventilation and other organ support.

\section{Acknowledgments}

Funding: None.

\section{Footnote}

Provenance and Peer Review: This article was commissioned by the Guest Editors (Jan Hau Lee, Vijay Srinivasan, and Debbie Long) for the series "Pediatric Critical Care" published in Translational Pediatrics. The article has undergone external peer review.

Reporting Checklist: The authors have completed the Narrative Review reporting checklist. Available at http:// dx.doi.org/10.21037/tp-21-5

Peer Review File: Available at http://dx.doi.org/10.21037/ tp-21-5

Conflicts of Interest: Both authors have completed the ICMJE uniform disclosure form (available at http://dx.doi. org/10.21037/tp-21-5). The series "Pediatric Critical Care" was commissioned by the editorial office without any funding or sponsorship. The authors have no other conflicts of interest to declare.

Ethical Statement: The authors are accountable for all 
aspects of the work in ensuring that questions related to the accuracy or integrity of any part of the work are appropriately investigated and resolved.

Open Access Statement: This is an Open Access article distributed in accordance with the Creative Commons Attribution-NonCommercial-NoDerivs 4.0 International License (CC BY-NC-ND 4.0), which permits the noncommercial replication and distribution of the article with the strict proviso that no changes or edits are made and the original work is properly cited (including links to both the formal publication through the relevant DOI and the license). See: https://creativecommons.org/licenses/by-nc-nd/4.0/.

\section{References}

1. Wolfsdorf JI, Glaser N, Agus M, et al. ISPAD Clinical Practice Consensus Guidelines 2018: Diabetic ketoacidosis and the hyperglycemic hyperosmolar state. Pediatr Diabetes 2018;19 Suppl 27:155-77.

2. Raghupathy P. Diabetic ketoacidosis in children and adolescents. Indian J Endocrinol Metab 2015;19:S55-7.

3. Dhatariya K. The evolution of DKA management. Br J Diabetes Vasc Dis 2015;15:31-3.

4. British Society for Paediatric Endocrinology and Diabetes. Interim guideline for the management of children and young people under the age of 18 years with diabetic ketoacidosis, 2020. [Accessed Jan 2021]. Available online: https://www.bsped.org.uk/media/1745/bsped-dkaguidelines-no-dka-link.pdf

5. National Institute for Health and Care Excellence. Diabetic ketoacidosis in children and young people, 2020. Available online: http://pathways.nice.org.uk/pathways/ diabetes-in-children-and-young-people

6. Tasker RC. Fluid management during diabetic ketoacidosis in children: guidelines, consensus, recommendations and clinical judgement. Arch Dis Child 2020;105:917-8.

7. Koves IH, Neutze J, Donath S, et al. The Accuracy of Clinical Assessment of Dehydration During Diabetic Ketoacidosis in Childhood. Diabetes Care 2004;27:2485-7.

8. Jayashree M, Williams V, Iyer R. Fluid Therapy For Pediatric Patients With Diabetic Ketoacidosis: Current Perspectives. Diabetes Metab Syndr Obes 2019;12:2355-61.

9. Ugale J, Mata A, Meert KL, et al. Measured degree of dehydration in children and adolescents with type 1 diabetic ketoacidosis. Pediatr Crit Care Med 2012;13:e103-7.
10. Poirier MP, Greer D, Satin-Smith M. A prospective study of the "two-bag system" in diabetic ketoacidosis management. Clin Pediatr (Phila) 2004;43:809-13.

11. Dhochak N, Jayashree M, Singhi S. A randomized controlled trial of one bag vs. two bag system of fluid delivery in children with diabetic ketoacidosis: Experience from a developing country. Journal of Critical Care 2018;43:340-5.

12. Kuppermann N, Ghetti S, Schunk JE, et al. Clinical Trial of Fluid Infusion Rates for Pediatric Diabetic Ketoacidosis. N Engl J Med 2018;378:2275-87.

13. Quilley CP, Lin YS, McGiff JC. Chloride anion concentration as a determinant of renal vascular responsiveness to vasoconstrictor agents. Br J Pharmacol 1993;108:106-10.

14. Mahler SA, Conrad SA, Wang H, et al. Resuscitation with balanced electrolyte solution prevents hyperchloremic metabolic acidosis in patients with diabetic ketoacidosis. Am J Emerg Med 2011;29:670-4.

15. Baalaaji M, Jayashree M, Nallasamy K, et al. Predictors and Outcome of Acute Kidney Injury in Children with Diabetic Ketoacidosis. Indian Pediatr 2018;55:311-4.

16. Williams V, Jayashree $M$, Nallasamy K, et al. 0.9\% saline versus Plasma-Lyte as initial fluid in children with diabetic ketoacidosis (SPinK trial): a double-blind randomized controlled trial. Critical Care 2020;24:1.

17. Yung M, Letton G, Keeley S. Controlled trial of Hartmann's solution versus $0.9 \%$ saline for diabetic ketoacidosis. J Paediatr Child Health 2017;53:12-7.

18. Rosenbloom AL. The management of diabetic ketoacidosis in children. Diabetes Ther 2010;1:103-20.

19. Bradley P, Tobias JD. Serum glucose changes during insulin therapy in pediatric patients with diabetic ketoacidosis. Am J Ther 2007;14:265-8.

20. Durward A, Ferguson LP, Taylor D, et al. The temporal relationship between glucose-corrected serum sodium and neurological status in severe diabetic ketoacidosis. Arch Dis Child 2011;96:50-7.

21. Al Hanshi S, Shann F. Insulin infused at 0.05 versus $0.1 \mathrm{units} / \mathrm{kg} / \mathrm{hr}$ in children admitted to intensive care with diabetic ketoacidosis. Pediatr Crit Care Med 2011;12:137-40.

22. Nallasamy K, Jayashree M, Singhi S, et al. Low-Dose vs Standard-Dose Insulin in Pediatric Diabetic Ketoacidosis: A Randomized Clinical Trial. JAMA Pediatrics 2014;168:999-1005.

23. Long B, Koyfman A. Emergency Medicine Myths: Cerebral Edema in Pediatric Diabetic Ketoacidosis and 
Intravenous Fluids. J Emerg Med 2017;53:212-21.

24. Kendir OT, Yilmaz HL, Ozkaya AK, et al. Determination of cerebral edema with serial measurement of optic nerve sheath diameter during treatment in children with diabetic ketoacidosis: a longitudinal study. J Pediatr Endocrinol Metab 2019;32:943-9.

25. Brown TB. Cerebral oedema in childhood diabetic ketoacidosis: Is treatment a factor? Emerg Med J 2004;21:141-4.

26. Eisenhut $M$. In diabetic ketoacidosis brain injury including cerebral oedema and infarction is caused by interleukin-1. Med Hypotheses 2018;121:44-6.

27. Karavanaki K, Karanika E, Georga S, et al. Cytokine response to diabetic ketoacidosis (DKA) in children with type 1 diabetes (T1DM). Endocr J 2011;58:1045-53.

28. Garro A, Chodobski A, Szmydynger-Chodobska J, et al. Circulating matrix metalloproteinases in children with diabetic ketoacidosis. Pediatr Diabetes 2017;18:95-102 .

29. Vavilala MS. Treating cerebral edema in diabetic ketoacidosis: caveats in extrapolating from traumatic brain injury*. Pediatr Crit Care Med 2013;14:723-4.

30. Dervan L, Poliakov A, Friedman SD, et al. Change

Cite this article as: Ravikumar N, Bansal A. Application of bench studies at the bedside to improve outcomes in the management of severe diabetic ketoacidosis in children-a narrative review. Transl Pediatr 2021;10(10):2792-2798. doi: $10.21037 /$ tp-21-5 in fractional anisotropy during treatment of diabetic ketoacidosis in children. Pediatr Res 2014;75:62-6.

31. Decourcey DD, Steil GM, Wypij D, et al. Increasing Use of Hypertonic Saline Over Mannitol in the Treatment of Symptomatic Cerebral Edema in Pediatric Diabetic Ketoacidosis: An 11-Year Retrospective Analysis of Mortality. Pediatr Crit Care Med 2013;14:694-700.

32. Tasker RC, Lutman D, Peters MJ. Hyperventilation in severe diabetic ketoacidosis. Pediatr Crit Care Med 2005;6:405-11.

33. Rameshkumar R, Bansal A, Singhi S, et al. Randomized Clinical Trial of 20\% Mannitol Versus 3\% Hypertonic Saline in Children With Raised Intracranial Pressure Due to Acute CNS Infections. Pediatr Crit Care Med 2020;21:1071-80.

34. Dhatariya K. Blood Ketones: Measurement, Interpretation, Limitations, and Utility in the Management of Diabetic Ketoacidosis. Rev Diabet Stud 2016;13:217-25.

35. Kurup PM, Rameshkumar R, Soundravally R, et al. Capillary versus Serum $\beta$-hydroxybutyrate in Pediatric Diabetic Ketoacidosis. Indian Pediatr 2019;56:126-9. 